\title{
Estimation of the burden of varicella in Europe before the introduction of universal childhood immunization
}

\author{
Margarita Riera-Montes ${ }^{1 *}$, Kaatje Bollaerts ${ }^{1} \mathbb{0}$, Ulrich Heininger ${ }^{2}$, Niel Hens ${ }^{3,4}$, Giovanni Gabutti ${ }^{5}$, Angel Gil ${ }^{6}$, \\ Bayad Nozad ${ }^{7}$, Grazina Mirinaviciute ${ }^{8}$, Elmira Flem ${ }^{8}$, Audrey Souverain', Thomas Verstraeten ${ }^{1}$ \\ and Susanne Hartwig ${ }^{10}$
}

\begin{abstract}
Background: Varicella is generally considered a mild disease. Disease burden is not well known and country-level estimation is challenging. As varicella disease is not notifiable, notification criteria and rates vary between countries. In general, existing surveillance systems do not capture cases that do not seek medical care, and most are affected by underreporting and underascertainment. We aimed to estimate the overall varicella disease burden in Europe to provide critical information to support decision-making regarding varicella vaccination.
\end{abstract}

Methods: We conducted a systematic literature review to identify all available epidemiological data on varicella lgG antibody seroprevalence, primary care and hospitalisation incidence, and mortality. We then developed methods to estimate age-specific varicella incidence and annual number of cases by different levels of severity (cases in the community, health care seekers in primary care and hospitals, and deaths) for all countries belonging to the European Medicines Agency (EMA) region and Switzerland.

Results: In the absence of universal varicella immunization, the burden of varicella would be substantial with a total of 5.5 million (95\% Cl: 4.7-6.4) varicella cases occurring annually across Europe. Variation exists between countries but overall the majority of cases (3 million; 95\% Cl: 2.7-3.3) would occur in children <5 years. Annually, 3-3.9 million patients would consult a primary care physician, 18,200-23,500 patients would be hospitalised, and 80 varicella-related deaths would occur (95\% Cl: 19-822).

Conclusions: Varicella disease burden is substantial. Most cases occur in children $<5$ years old but adults require hospitalisation more often and are at higher risk of death. This information should be considered when planning and evaluating varicella control strategies. A better understanding of the driving factors of country-specific differences in varicella transmission and health care utilization is needed. Improving and standardizing varicella surveillance in Europe, as initiated by the European Centre for Disease Prevention and Control (ECDC), is important to improve data quality to facilitate inter-country comparison.

Keywords: Varicella, Disease burden, Europe

\footnotetext{
* Correspondence: margarita.riera@p-95.com

'P95 Pharmacovigilance and Epidemiology Services, Koning Leopold III Laan

1, 3001 Leuven, Belgium

Full list of author information is available at the end of the article
} International License (http://creativecommons.org/licenses/by/4.0/), which permits unrestricted use, distribution, and reproduction in any medium, provided you give appropriate credit to the original author(s) and the source, provide a link to the Creative Commons license, and indicate if changes were made. The Creative Commons Public Domain Dedication waiver (http://creativecommons.org/publicdomain/zero/1.0/) applies to the data made available in this article, unless otherwise stated. 


\section{Background}

Varicella Zoster Virus (VZV) is a double-stranded DNA virus of the herpes virus family [1]. It causes varicella (chickenpox), a highly communicable disease which is usually contracted in early childhood, typically affecting children 2-8 years of age [1]. Varicella is usually a mild disease, but can cause complications requiring hospitalisation [2,3] and, in rare instances, can even be fatal [4]. After initial infection with VZV, the virus becomes latent in sensory nerve ganglia. Viral reactivation, which usually occurs with increased age or immunosuppression, causes herpes zoster (shingles). Shingles is a painful condition associated with complications including postherpetic neuralgia and cerebrovascular disease [1].

Although several vaccines for the prevention of varicella are licensed in the European Union (EU), few EU member states $(n=7)$ have implemented a general recommendation for their use [4]. This may be related to a lack of data on the epidemiology of varicella at the country level. The estimation of varicella burden at country level is challenging. Varicella is not a mandatory reportable disease in the EU [4], and systematic pan-European surveillance does not exist. Data, if existing, are based either on national mandatory reporting or more rarely, on national sentinel surveillance systems [5]. The systems differ by the type of cases captured (all cases vs. medically attended cases or only cases with complications), case definitions used, methods for case ascertainment (clinical, laboratory, epidemiologically-linked, or combinations thereof), and data type (case-based or aggregated data). Additionally, available surveillance systems are almost all affected by underreporting $[6,7]$ and underascertainment: most surveillance systems only capture medically attended disease but not all patients with varicella seek medical care [8].

Systematic literature reviews (SLRs) on the burden of varicella in the EU have recently been conducted by ECDC [4] and Helmuth et al. [3], but like previous reviews, they were descriptive in nature. We set out to quantify the country-specific burden of varicella disease in Europe by using all publicly available data and extrapolating for those countries where we did not find data. To our knowledge, our study is the first to systematically estimate the burden of varicella for individual European countries. We anticipate that this work will contribute to a better understanding of the burden of varicella in Europe, and support decision-making regarding varicella vaccination.

\section{Methods}

Systematic literature review Search strategy

A PubMed search was conducted for peer-reviewed publications reporting primary incidence, mortality or seroprevalence data in any language in countries under the European Medicines Agency (EMA) plus Swizerland. The search was limited to articles published on or after January 1st, 1995 and restricted to human studies. The full search string used was "Varicella AND (mortality OR complications OR epidemiology OR seroprevalence OR prevalence OR incidence) AND ("Europe"[Mesh] OR Austria OR Belgium OR Bulgaria OR Croatia OR Cyprus OR "Czech Republic" OR Denmark OR Estonia OR Finland OR France OR Germany OR Greece OR Hungary OR Iceland OR Ireland OR Italy OR Latvia OR Liechtenstein OR Lithuania OR Luxembourg OR Malta OR Netherlands OR Norway OR Poland OR Portugal OR Romania OR Slovenia OR Slovakia OR Spain OR Sweden OR "United Kingdom" OR Switzerland)". Additional information was obtained from the ECDC and national health institutes websites, and through personal communication with national varicella surveillance focal points. Hand searching of the reference lists of papers selected for inclusion was conducted to identify additional publications.

\section{Outcomes}

Outcomes of interest for the literature review were varicella IgG antibody seroprevalence, incidence of varicella (varicella associated primary care visits and hospitalisations) and mortality.

\section{Eligibility criteria}

Studies were eligible for inclusion if: 1) they provided data for one or more of the outcomes of interest in the general population, 2) the data were collected before the introduction of universal varicella immunization for countries where universal varicella immunization has been introduced, and 3) the study was published on or after January 1st 1995. Studies were excluded if they did not contain primary data or if the study population was not representative of the general population in terms of varicella transmission dynamics and/or risk of infection (e.g. immunosuppressed patients, imprisoned individuals, or day care workers).

\section{Data extraction}

Two reviewers (MB, MR) screened titles and abstracts. Discrepancies were extensively discussed and no third reviewer was necessary to resolve disagreements. Evaluation of full text eligibility and data extraction was conducted by a single reviewer (MR). For articles published in languages other than English, reviewers were able to directly read and extract articles in Dutch, German, French, Swedish, Italian, Portuguese and Spanish. For one article in Icelandic, translation software was used for the body text. Legends for the tables and the abstract were provided in English by the journal. For quality control, a sample of $10 \%$ of the papers was re-extracted by a 
third reviewer (TV). The following data was extracted and stored in an MS Excel grid when available: author, journal, year of publication, country, study design, setting (community, primary care, hospital, other), population, case ascertainment, age range, sample size, and incidence or proportion with $95 \%$ confidence intervals (CIs). The quality of the evidence was assessed by a single reviewer (MR) with a risk of bias tool adapted from the one by Hoy et al. [9] (Additional file 1). This tool facilitated the scoring of studies from 0 to 8 , with the following four categorisations: Excellent (very low risk of bias) - score 8 and prospective study design; Good (low risk of bias) - score 8 , but no prospective study design; Acceptable (medium risk of bias) score 6-7; Low (high risk of bias) - score less than 6 .

\section{Incidence estimation}

We aimed to estimate the annual incidence rates of varicella cases that 1 ) occurred in the community (with or without health care visit), 2) resulted in an ambulatory primary health care visit, 3) required hospitalisation, or 4) caused death.

Data sources were used for incidence estimation if: 1) studies attained a quality score of 6 or more; 2) data collection was conducted for at least 1 year; and 3) agespecific data was provided. In cases where the same data source was used to report incidence for different time periods, we selected the source with the longest time period covered and/or most recent data for inclusion in our analyses. We excluded data derived from mandatory notification systems which may be substantially affected by underreporting [10], except for countries where mandatory notification was the only data source available. When the age groups reported in the original data source did not match our age groups of interest, we either used weighted averages (to combine several age groups) or we redistributed the age groups assuming constant incidence within the age groups (to split a single age group). Age groups without upper limits were excluded from the calculations, because the width of the age group was not known and therefore the incidence cannot be recalculated for the age group of interest.

\section{Incidence estimation for countries with data}

Few studies in Europe have assessed the incidence rate of varicella at the community level. We therefore opted to derive the annual age-specific varicella incidence (per 100,000) from seroprevalence studies, which are commonly available. Particularly, for each country we first estimated age-specific seroprevalence using the catalytic model with a piecewise constant force of infection. Then, from these estimated profiles, we derivedincidence rates (and 95\% CIs) as differences in seroprevalences for six age groups, < 5, 5-9, 10-14, 15-19, 20-39 and $40+$ years. This approach assumes lifelong immunity, time homogeneity and non-differential mortality. For more details on the methodology used, we refer to Bollaerts et al. (Bollaerts K, Riera-Montes M, Hens N et al. A systematic review of varicella seroprevalence in European countries before universal childhood immunization: deriving incidence from seroprevalence data. Submitted 2017).

For incidence rates at the primary care and hospital level, we relied upon the published age-specific data for countries with more extensive information. For countries with more than one estimate, we provide a range with the lowest and highest estimates.

Varicella-specific mortality data was obtained from the World Health Organisation (WHO) European Detailed Mortality Database (DMDB) [11]. The DMDB contains mortality data by cause of death (ICD-9 or ICD-10 codes), age and sex. Other country-specific mortality data sources identified during the SLR were found either to rely upon the same data source that feeds into the WHO DMDB or to provide comparable results. We obtained mortality data for all countries for the 10 most recent years available prior to the introduction of universal varicella immunization. We calculated the Poisson exact $95 \%$ CIs of the mortality rates.

\section{Incidence estimation for countries without data}

To obtain age-specific community, primary care and hospital incidence estimates for countries without data, we took a 2-step approach. Firstly, we tried to build prediction models for each outcome and age group, calibrated using information from the countries with data. Secondly, when the model failed to predict (i.e. non-significance of any of the potential predictor variables), we extrapolated the minimum and maximum observed estimates within the same age group from countries with data (Table 2).

Particularly, we built a linear regression model based on the incidences in the younger age groups and countrylevel prediction variables that have been shown to be associated with country-level differences in varicella transmissibility and health care use [12,13] (Additional file 2): proportion of children $<3$ years that receive no formal childcare [14], population density [14], inequality in income distribution [14], proportion of people at risk of poverty [14], total health expenditure [15], proportion of households with 1, 2, 3, and 4 or more children [14], number of annual consultations of a medical doctor per inhabitant [14], and number of acute hospital discharges per 100 population [14].

We failed to predict primary care incidence (PCI) and hospitalisation incidence (HI). Therefore, we relied upon the ratio between PCI and community incidence - the primary care rate $(\mathrm{PCR})$, and on the ratio between $\mathrm{HI}$ and community incidence - the hospitalisation rate (HR). 
Specifically, we calculated PCI or HI by multiplying the country's age-specific community incidence with the corresponding age-specific average minimum-maximum observed PCR or HR. We preferred this approach to the alternative approach of simply imputing the PCI and $\mathrm{HI}$ that was observed in other countries, as this would not take into account differences in community incidence.

No imputations were required for mortality data, as these were available from the DMDB for all countries.

\section{Validation}

To evaluate our methodology, we used information from a recent varicella study in Norway [16], which was conducted in 2015 and published after our literature search had concluded. This study provided both age-specific seroprevalence proportions and age-specific estimates of $\mathrm{PCI}$ at the national level. We used this published seroprevalence data to estimate varicella community incidence and derive PCI as described above.

\section{Estimation of annual number of cases}

To estimate the annual country-specific numbers of varicella-associated community cases, ambulatory primary care consultations, hospitalisations, and deaths per age group, we applied age-specific community incidence, PCI, HI, and mortality rates to each country's population. Population data was obtained from Eurostat for the latest year available (2015) [14]. The total annual number of cases within Europe was then calculated by summing the number of cases for each country. The numbers of varicella-associated community cases and deaths are presented as point estimates with $95 \%$ CIs, while the numbers of varicella-related primary care consultations and hospitalisations are presented as a range.

We compared the estimated annual country-specific numbers of varicella-associated community cases to the numbers reported to EUVAC.NET, in order to estimate the underreporting of the disease. EUVAC.NET is a European surveillance network for selected vaccinepreventable diseases that was active until 2011 and was hosted by the Staten Serum Institute (SSI), Denmark. It incorporated 18 countries; all EU Member States up to 2011, as well as Croatia, Iceland, Norway, Switzerland and Turkey. Number of varicella cases were reported annually by all countries. We compared the EUVAC.NET data of 2009-2010 to our results, and calculated the ratio of reported to estimated number of cases, expressed in percentages.

\section{Results}

\section{Systematic literature review}

The literature search was conducted in PubMed on October 2nd 2015. Identification of grey literature sources was conducted between October and December 2015.
We identified 120 data sources from 31 countries for extraction (Fig. 1) [5, 7, 8, 11, 17-127]. Most data sources (97/120) scored the maximum in terms of quality assessment (score of 8). Only one data source scored less than 6 . A table summarizing the main characteristics of all selected data sources, and a summarised version of the full data extraction table is provided in Additional files 3 and 4 .

The most frequently found unique data source was on seroprevalence $(n=52)$ [17-68], followed by hospitalisation $(n=39)[23,27,40,69-104]$, primary care $(n=27)$ [7, 8, 23, 27, 74, 76, 78-81, 97, 99, 102, 104-117], mortality $(n=15)[11,23,27,74-76,79,80,84,91,97,118$ 121], and incidence of reported varicella cases through mandatory surveillance $(n=14)$ (Table 1$)[5,26,27,40$, $79,80,101,116,122-127]$. Varicella community incidence was estimated from 43 seroprevalence data sources from 16 countries [17-27, 29-41, 44-47, 49-54, $57-62,64,65,68]$, primary care incidence from $17 \mathrm{PCI}$ data sources from 8 countries $[23,74,76,79,80,99$, $101,102,104-106,109,110,112,114,124,126]$, hospitalisation incidence from $18 \mathrm{HI}$ data sources from 10 countries $[23,69-73,75,76,79-81,84,87,90,91,94$, $102,103]$, and one data source was used to estimate mortality in 31 countries [11] (Table 2). Data on all four outcomes was only available for six countries (Belgium, France, Italy, the Netherlands, Spain and the UK).

\section{Varicella community incidence}

The final model to predict community incidence in $<5$ year olds included as country-level predictors the proportion of children $<3$ years attending pre-school care and population density, with a moderate goodness of fit $\left(R^{2}=40 \%\right)$. For 5-9 year olds, the prediction model included the incidence in $<5$ year olds with a moderate to high goodness of fit $\left(R^{2}=80 \%\right)$. For the older age groups, we had to rely upon extrapolation. We first categorised countries based on the speed of varicella acquisition in children $<5$ years (annual incidence $<$ or $\geq 10 \%$ ) as in Bollaerts et al. (Bollaerts K, Riera-Montes $\mathrm{M}$, Hens $\mathrm{N}$ et al. A systematic review of varicella seroprevalence in European countries before universal childhood immunization: deriving incidence from seroprevalence data. Submitted 2017). Then, we extrapolated the minimum and maximum age specific community incidence observed in countries with data (Table 2) to countries without data within the same category.

Age-specific annual incidence rates of varicella, as derived from serological data, varied considerably across the countries, particularly in age groups $10-14$ and 1519 years. In most countries the highest annual incidence was observed amongst children aged $<5$ years, ranging from 7052 (Greece) to 17,974 (Malta) per 100,000 (Table 


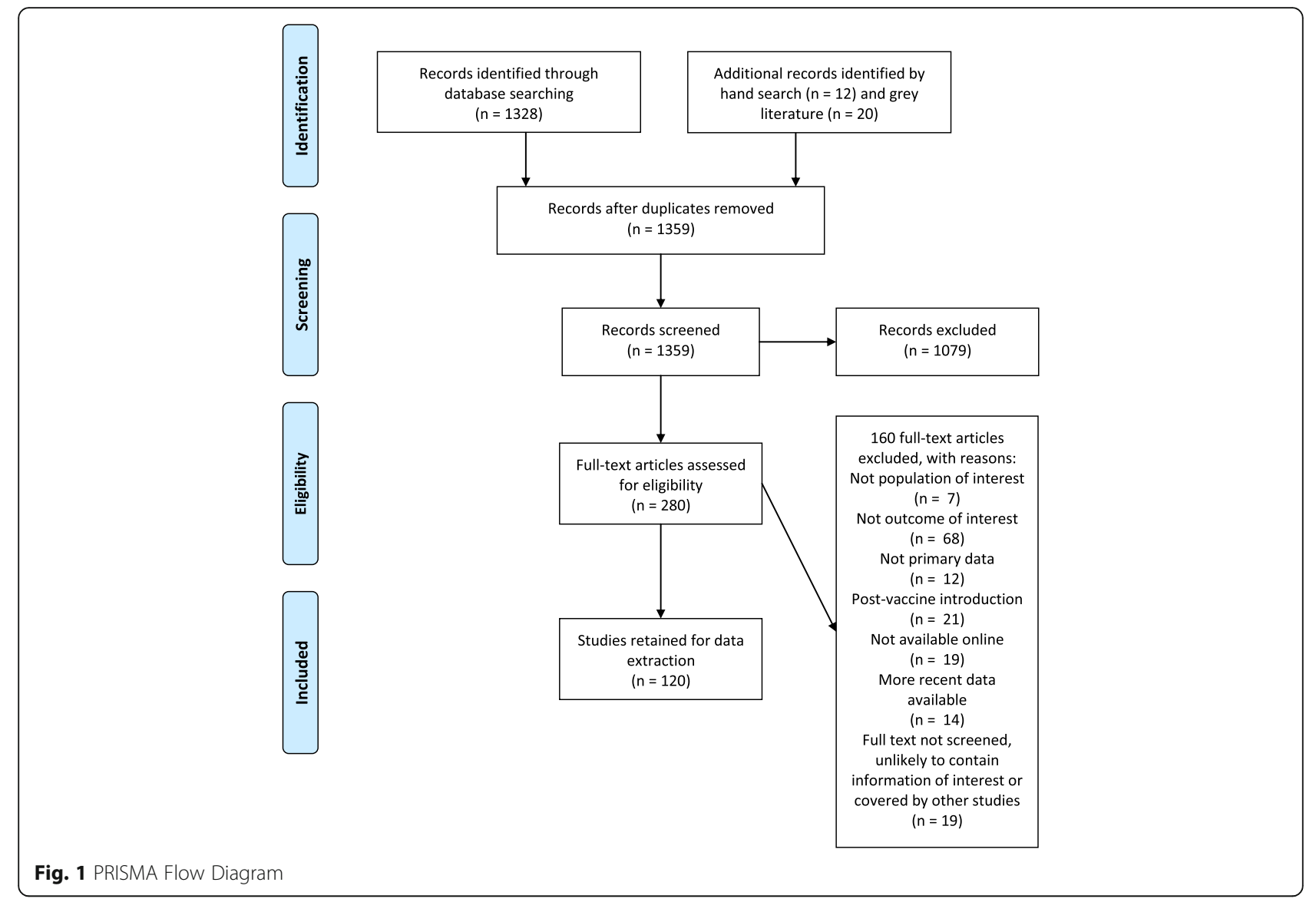

3). In Bulgaria, Czech Republic, Italy, Romania, Switzerland and Greece the highest incidence was observed in 5-9 year olds. From the age of 10 years onwards, varicella incidence dropped drastically. Countries with the highest incidence in children $<5$ years had lower incidence rates in 10 14 year olds than those countries with the highest incidence in children 5-9 years of age. By the age of $15-19$ years, incidence was $<1000$ per 100,000 in all countries with the exception of Greece.

\section{Varicella primary care incidence}

We failed to model the PCI for any of the age groups and we therefore had to rely upon the maximumminimum approach. The estimated PCRs ranged from $18 \%$ (Netherlands, 5-9 year olds) to $100 \%$ (Netherlands, 10-64 y; Italy, 10-14 y; Spain, 10-14 y; UK, 10-39 y; France, 5-64 y) across all age groups.

Varicella primary care incidence was highest in children $<5$ years with substantial inter-country variation, ranging from 1100 per 100,000 in Romania to 13,069 per 100,000 in France. Incidence decreased with age, dropping substantially from the age of 10 years onwards (Table 4).

\section{Varicella hospitalisation incidence}

We also failed to model the HI for any age group and used the minimum-maximum approach instead. The estimated HRs ranged from $0.05 \%$ (Ireland, 5-9 y) to 3.5\% (Netherlands, 20-39 y) across all age groups.

All countries presented the highest $\mathrm{HI}$ in children $<5$ years. Incidence ranged from 9 per 100,000 in Sweden to 75 per 100,000 in France, decreasing to $<20$ per 100,000 for all countries in the 5-9 years age group (Table 5). In older age groups, HI remained $\leq 10$ per 100,000.

\section{Varicella mortality}

Estimated varicella mortality was very low with an annual incidence $<0.2$ deaths per 100,000 in all age groups and countries (Table 6). For most countries, the mortality was highest in children $<5$ years. However, in some countries (Czech Republic, Greece, Ireland, Slovakia), mortality was higher in the 5-9 year age group, and in Lithuania, the mortality peak was found in the 10-14 years age group.

\section{Validation}

The observed annual community incidence in children under 10 years of age in Norway was in line with our 
Table 1 Number of literature sources available per outcome per country, broken down by outcome ${ }^{a}$

\begin{tabular}{|c|c|c|c|c|c|c|}
\hline \multirow{2}{*}{$\begin{array}{l}\text { Country } \\
\text { Country }\end{array}$} & \multicolumn{2}{|c|}{$\mathrm{N}$ of sources } & \multicolumn{4}{|l|}{ Outcome } \\
\hline & $\mathrm{N}$ papers & $\mathrm{N}$ grey literature & Sero-prevalence & Primary care incidence & Hospitalization incidence & Mortality \\
\hline Austria & 1 & 1 & 1 & & & 1 \\
\hline Belgium & 5 & 4 & 4 & 3 & 2 & 3 \\
\hline Bulgaria & 0 & 4 & & 3 & & 1 \\
\hline Croatia & 1 & 4 & 1 & 3 & & 1 \\
\hline Cyprus & 0 & 3 & & 2 & & 1 \\
\hline Czech Rep & 0 & 4 & & 3 & & 1 \\
\hline Denmark & 0 & 1 & & & & 1 \\
\hline Estonia & 0 & 4 & & 3 & & 1 \\
\hline Finland & 4 & 4 & 3 & 3 & & 2 \\
\hline France & 6 & 7 & 3 & 6 & 3 & 4 \\
\hline Germany & 7 & 1 & 2 & 2 & 2 & 2 \\
\hline Greece & 4 & 2 & 1 & 1 & 3 & 1 \\
\hline Hungary & 1 & 4 & & 4 & & 1 \\
\hline Iceland & 1 & 1 & 1 & & & 1 \\
\hline Ireland & 4 & 4 & 3 & & 3 & 2 \\
\hline Italy & 14 & 3 & 7 & 9 & 5 & 2 \\
\hline Latvia & 0 & 3 & & 2 & & 1 \\
\hline Lithuania & 0 & 4 & & 3 & & 1 \\
\hline Luxembourg & 1 & 1 & 1 & 1 & 1 & 1 \\
\hline Malta & 0 & 4 & & 3 & & 1 \\
\hline Netherlands & 7 & 2 & 4 & 5 & 4 & 2 \\
\hline Norway & 0 & 1 & & & & 1 \\
\hline Poland & 3 & 4 & 1 & 5 & 1 & 1 \\
\hline Portugal & 3 & 1 & 2 & 1 & & 1 \\
\hline Romania & 1 & 4 & & 4 & & 1 \\
\hline Slovakia & 1 & 4 & 1 & 3 & & 1 \\
\hline Slovenia & 2 & 4 & 1 & 4 & & 1 \\
\hline Spain & 22 & 2 & 10 & 5 & 9 & 3 \\
\hline Sweden & 4 & 1 & 1 & & 3 & 1 \\
\hline Switzerland & 6 & 1 & 5 & 1 & & 1 \\
\hline UK & 13 & 4 & 5 & 12 & 3 & 4 \\
\hline Total & 101 & 18 & 57 & 91 & 39 & 46 \\
\hline Unique data sources & & & 52 & 37 & 39 & 15 \\
\hline
\end{tabular}

${ }^{a}$ This includes literature sources that were not eligible for the estimation of the burden of varicella disease in Europe

Table 2 Number of data sources and countries included in incidence estimation per outcome

\begin{tabular}{llll}
\hline Outcome & N data sources & N countries & Countries with data \\
\hline $\begin{array}{l}\text { Community incidence (derived } \\
\text { from seroprevalence data) }\end{array}$ & 43 & 16 & Belgium, Finland, France, Germany, Greece, Iceland, Ireland, Italy, Luxembourg, \\
Primary care incidence & 17 & & Netherlands, Poland, Slovakia, Slovenia, Spain, Switzerland, UK \\
Hospitalization incidence & 18 & 8 & Belgium, France, Italy, Netherlands, Poland, Romania, Spain, UK \\
Mortality & 1 & 10 & Belgium, France, Germany, Greece, Ireland, Italy, Netherlands, Spain, Sweden, UK \\
\hline
\end{tabular}


Table 3 Age-specific annual community incidence $(/ 100,000)$ of varicella in European countries before the introduction of universal childhood immunization programs

\begin{tabular}{|c|c|c|c|c|c|c|}
\hline \multirow[t]{2}{*}{ Country } & \multicolumn{6}{|c|}{ Annual incidence $/ 100.000(95 \% \mathrm{Cl})$ per age group in years } \\
\hline & $<5$ & $5-9$ & $10-14$ & $15-19$ & $20-39$ & $40+$ \\
\hline$\overline{\text { Austria }^{a}}$ & $8986(7449-10,523)$ & $8421(7643-9200)$ & $1267(882-1652)$ & $688(554-822)$ & $195(192-198)$ & $18(10-26)$ \\
\hline Belgium & $14,628(13,848-15,408)$ & $4126(3160-5124)$ & $284(136-480)$ & $220(114-342)$ & $120(75-163)$ & $38(31-45)$ \\
\hline Bulgaria $^{a}$ & $8097(6237-9957)$ & $9053(8136-9970)$ & $1267(882-1652)$ & $688(554-822)$ & $195(192-198)$ & $18(10-26)$ \\
\hline Croatia $^{a}$ & $8911(7386-10,436)$ & $8475(7685-9264)$ & $1267(882-1652)$ & $688(554-822)$ & $195(192-198)$ & $18(10-26)$ \\
\hline Cyprus $^{\mathrm{a}}$ & $10,039(8838-11,240)$ & 7674 (7022-8325) & $272(0-916)$ & $213(0-698)$ & $123(0-372)$ & $48(0-113)$ \\
\hline Czech Republic $^{a}$ & $7707(5350-10,065)$ & $9330(8346-10,313)$ & $1267(882-1652)$ & $688(554-822)$ & 195 (192-198) & $18(10-26)$ \\
\hline Denmark $^{a}$ & $15,965(12,545-19,385)$ & $3464(2307-4622)$ & $272(0-916)$ & $213(0-698)$ & $123(0-372)$ & $48(0-113)$ \\
\hline Estonia $^{a}$ & $8850(7256-10,444)$ & 8518 (7720-9317) & $1267(882-1652)$ & $688(554-822)$ & 195 (192-198) & $18(10-26)$ \\
\hline Finland & $10,130(9336-10,850)$ & $8680(7830-9530)$ & $40(0-102)$ & $38(0-94)$ & $35(0-79)$ & $30(0-55)$ \\
\hline France & $13,488(12,698-14,254)$ & $4668(3554-5722)$ & $554(260-974)$ & 388 (208-596) & $172(113-222)$ & $36(17-56)$ \\
\hline Germany & $11,884(10,972-12,646)$ & 7048 (6192-8046) & $246(156-374)$ & $190(126-266)$ & $103(77-128)$ & $32(24-40)$ \\
\hline Greece & 7052 (5986-7998) & 7462 (5026-9578) & $2804(0-6364)$ & $1370(0-1666)$ & $310(0-543)$ & $14(0-213)$ \\
\hline Hungary $^{a}$ & $8752(7101-10,404)$ & $8588(7775-9401)$ & $1267(882-1652)$ & $688(554-822)$ & 195 (192-198) & $18(10-26)$ \\
\hline Iceland & $11,460(8400-12,520)$ & $7940(6840-11,260)$ & $0(0-0)$ & $0(0-0)$ & $0(0-0)$ & $0(0-0)$ \\
\hline Ireland & $11,954(10,688-13,194)$ & $6434(4894-7776)$ & $76(0-412)$ & $72(0-334)$ & $65(0-209)$ & $52(0-91)$ \\
\hline Italy & $8020(7320-8736)$ & 8118 (7072-9112) & $916(666-1242)$ & $698(534-892)$ & $372(320-421)$ & $113(93-128$ \\
\hline Latvia $^{a}$ & $9239(7726-10,751)$ & $8242(7498-8986)$ & $1267(882-1652)$ & $688(554-822)$ & 195 (192-198) & $18(10-26)$ \\
\hline Lithuania $^{a}$ & $9468(8045-10,891)$ & $8079(7365-8794)$ & $1267(882-1652)$ & $688(554-822)$ & $195(192-198)$ & $18(10-26)$ \\
\hline Luxembourg & $15,720(14,482-16,790)$ & $3292(2070-4560)$ & $152(58-314)$ & $128(54-238)$ & $86(44-126)$ & $41(28-49)$ \\
\hline Malta ${ }^{a}$ & $17,974(7481-28,467)$ & $2037(489-3586)$ & $272(0-916)$ & $213(0-698)$ & $123(0-372)$ & $48(0-113)$ \\
\hline Netherlands & $16,122(15610-16,592)$ & $3350(2854-3902)$ & $20(4-46)$ & $20(4-42)$ & $18(4-36)$ & $15(3-26)$ \\
\hline Norway ${ }^{a}$ & $13,279(10,433-16,124)$ & $5372(4653-6092)$ & $272(0-916)$ & $213(0-698)$ & $123(0-372)$ & $48(0-113)$ \\
\hline Poland & 8974 (7980-9990) & 7734 (6148-9192) & $1652(662-2740)$ & $822(492-1022)$ & 192 (94-284) & $10(1-71)$ \\
\hline Portugal $^{\mathrm{a}}$ & $12,693(11,009-14,378)$ & $5788(5134-6443)$ & $272(0-916)$ & $213(0-698)$ & $123(0-372)$ & $48(0-113)$ \\
\hline Romania $^{a}$ & 7108 (4663-9552) & $9755(8665-10,846)$ & $1267(882-1652)$ & $688(554-822)$ & $195(192-198)$ & $18(10-26)$ \\
\hline Slovakia & $9362(8398-10,330)$ & $8264(6926-9564)$ & $882(462-1494)$ & $554(348-758)$ & $198(152-230)$ & $26(8-56)$ \\
\hline Slovenia & $11,640(10,764-12,472)$ & $6954(5978-7956)$ & $274(148-464)$ & $220(128-336)$ & $132(93-169)$ & $51(36-61)$ \\
\hline Spain & $10,874(10,234-11,550)$ & $7312(6572-8014)$ & $314(236-400)$ & $260(202-318)$ & 165 (139-188) & $71(64-76)$ \\
\hline Sweden ${ }^{a}$ & $13,578(10,635-16,522)$ & $5160(4401-5919)$ & $272(0-916)$ & $213(0-698)$ & $123(0-372)$ & $48(0-113)$ \\
\hline Switzerland & 7368 (6494-8314) & $11,798(10,816-12,722)$ & $74(0-346)$ & $68(0-220)$ & $54(0-94)$ & $36(0-42)$ \\
\hline UK & $12,982(12,230-13,718)$ & $4656(3638-5610)$ & $388(160-696)$ & $324(148-530)$ & $212(120-281)$ & $95(74-106)$ \\
\hline
\end{tabular}

${ }^{\mathrm{a}}$ countries where community incidence was predicted

predictions (8669 vs 9326 per 100,000). However, the model overestimated the number of cases in $<5$ year olds and underestimated the incidence in 5-9 year olds compared to the observed data (Table 7). The model also predicted substantially higher estimates of varicella PCI in Norway in most age groups compared to observed data, particularly in children under 10 years of age (Table 8).

\section{Annual number of varicella cases}

We estimated that across European countries, and in the absence of universal varicella immunization, 5.5 million (95\% CI: 4.7-6.4) new varicella cases would occur annually. Most cases (3 million; 95\% CI: 2.7-3.3) would occur in children $<5$ years. At least $54 \%$ of varicella cases are expected to result in an ambulatory primary care visit and $0.3 \%$ will require hospitalisation (Table 9), implying that annually 3-3.9 million patients would consult a primary care physician and 18,200-23,500 patients be hospitalised. In addition, approximately 80 varicellarelated deaths are expected to occur every year (95\% CI: 19-822) (Fig. 2, Table 9). (See Additional file 5 for country-specific data).

From comparing the number of annual varicella cases reported to EUVAC.NET to our estimates, an important 
Table 4 Age-specific annual primary care incidence/100,000 of varicella in Europe before the introduction of universal childhood immunization programs

\begin{tabular}{|c|c|c|c|c|c|c|}
\hline \multirow[t]{2}{*}{ Country } & \multicolumn{6}{|c|}{ Annual incidence $/ 100,000$ (min-max) per age group in years } \\
\hline & $<5$ & $5-9$ & $10-14$ & $15-19$ & $20-39$ & $40+$ \\
\hline$\overline{\text { Austria }^{a}}$ & $4773-5322$ & $3623-5076$ & $1610-3457$ & $454-982$ & $136-251$ & $11-18$ \\
\hline Belgium & 4502-NA & 1006-NA & $190-N A$ & 63-NA & 49-NA & $15-N A$ \\
\hline Bulgaria $^{a}$ & $4301-4796$ & $3895-5457$ & $1610-3457$ & 454-982 & $136-251$ & $11-18$ \\
\hline Croatia $^{a}$ & $4733-5278$ & $3646-5109$ & $1610-3457$ & 454-982 & $136-251$ & $11-18$ \\
\hline Cyprus $^{a}$ & $5332-5946$ & $3302-4626$ & $346-742$ & $141-304$ & $86-158$ & $30-49$ \\
\hline Czech Rep. ${ }^{a}$ & 4093-4565 & 4014-5624 & $1610-3457$ & 454-982 & $136-251$ & $11-18$ \\
\hline Denmark $^{a}$ & $8480-9456$ & $1490-2088$ & $346-742$ & $141-304$ & $86-158$ & $30-49$ \\
\hline Estonia $^{a}$ & $4701-5242$ & $3665-5134$ & $1610-3457$ & 454-982 & $136-251$ & $11-18$ \\
\hline Finland $^{a}$ & $5380-6000$ & $3735-5232$ & $51-109$ & $25-54$ & $24-45$ & $19-31$ \\
\hline France & $10,694-13,069$ & $3344-5917$ & $368-1283$ & $161-342$ & $68-281$ & $28-56$ \\
\hline Germany $^{a}$ & $6312-7039$ & $3032-4248$ & $313-671$ & $125-271$ & $72-133$ & $20-33$ \\
\hline Greece $^{a}$ & $3746-4177$ & $3211-4498$ & $3562-7651$ & 904-1955 & 216-398 & $9-15$ \\
\hline Hungary $^{a}$ & $4649-5184$ & $3695-5177$ & $1610-3457$ & 454-982 & $136-251$ & $11-18$ \\
\hline Iceland ${ }^{a}$ & $6087-6788$ & $3416-4786$ & $0-0$ & $0-0$ & $0-0$ & $0-0$ \\
\hline Ireland ${ }^{a}$ & $6349-7080$ & $2768-3878$ & $97-207$ & 48-103 & $45-83$ & $33-53$ \\
\hline Italy & $7476-7582$ & $4548-5062$ & $1052-2283$ & 461-996 & $260-478$ & $71-116$ \\
\hline Latvia $^{a}$ & $4907-5472$ & $3546-4968$ & $1610-3457$ & 454-982 & $136-251$ & $11-18$ \\
\hline Lithuania $^{a}$ & 5029-5608 & $3476-4870$ & $1610-3457$ & 454-982 & $136-251$ & $11-18$ \\
\hline Luxembourg $^{a}$ & $8350-9311$ & 1416-1984 & 193-415 & 84-183 & $60-111$ & $26-42$ \\
\hline Malta ${ }^{a}$ & $9547-10,646$ & $876-1228$ & $346-742$ & $141-304$ & $86-158$ & $30-49$ \\
\hline Netherlands & $3032-5817$ & $591-1535$ & $52-180$ & $32-92$ & $23-50$ & $12-23$ \\
\hline Norway $^{a}$ & 7053-7865 & $2311-3238$ & $346-742$ & $141-304$ & $86-158$ & $30-49$ \\
\hline Poland & 3929-NA & 4106-NA & 923-NA & $197-N A$ & 77-NA & 9-NA \\
\hline Portugal $\left.\right|^{a}$ & $6742-7518$ & $2490-3489$ & $346-742$ & $141-304$ & 86-158 & $30-49$ \\
\hline Romania & 1100-NA & 1450-NA & 1000-NA & 500-NA & 89-NA & 8-NA \\
\hline Slovakia ${ }^{a}$ & 4973-5545 & $3556-4981$ & $1121-2407$ & 366-791 & 138-255 & $17-27$ \\
\hline Slovenia ${ }^{a}$ & $6182-6894$ & 2992-4192 & $348-748$ & $145-314$ & $92-170$ & $32-52$ \\
\hline Spain & 8304-NA & 3281-NA & 747-NA & $172-371$ & $115-212$ & $45-73$ \\
\hline Sweden ${ }^{a}$ & $7212-8042$ & $2220-3110$ & $346-742$ & $141-304$ & 86-158 & $30-49$ \\
\hline Switzerland $^{\mathrm{a}}$ & $3913-4364$ & $5076-7112$ & $94-202$ & $45-97$ & $38-69$ & $22-37$ \\
\hline UK & 3838-4695 & $1562-3012$ & 339-653 & $245-365$ & 213-255 & $22-67$ \\
\hline
\end{tabular}

${ }^{a}$ countries where primary care incidence was predicted NA: Not applicable

under-reporting to EUVAC.NET was found, with only $<1 \%$ (Greece: 6 vs 109,214 cases) to 51\% (Slovenia: 11,074 vs 21,729 cases) of all cases of varicella occurring in the community reported to EUVAC.NET (Table 10) [123].

\section{Discussion}

We estimate that in the absence of universal varicella immunization, a total of 5.5 million (95\% CI: 4.7-6.4) varicella cases would occur annually across Europe. It has previously been estimated that the annual number of new varicella cases in a country correspond approximately to the size of its birth cohort $[4,27,94,128]$.
Given that according to Eurostat [14] there were 5.2 million live births in Europe in 2015, this is in line with our estimates. Our study estimates that more than half of all varicella cases occur in children $<5$ years of age, as has been reported previously [4].

We found that community incidence varied greatly between countries, particularly in children and adolescents. This probably reflects different country-specific dynamics in varicella transmission during childhood, which have been associated with differences in social mixing patterns $[12,13]$. Countries with low incidence rates in children $<5$ years of age have higher incidence rates in 
Table 5 Age-specific annual hospitalization incidence $(/ 100,000)$ of varicella in Europe before the introduction of universal childhood immunization programs

\begin{tabular}{|c|c|c|c|c|c|c|}
\hline \multirow[t]{2}{*}{ Country } & \multicolumn{6}{|c|}{ Annual incidence /100,000 (min-max) per age group in years } \\
\hline & $<5$ & $5-9$ & $10-14$ & $15-19$ & $20-39$ & $40+$ \\
\hline Austria $^{a}$ & $28-34$ & $10-13$ & $6-9$ & $3-5$ & $3-3$ & $0-0$ \\
\hline Belgium & 79-NA & 3-NA & $1-2$ & $1-2$ & $2-2$ & $0-1$ \\
\hline Bulgaria $^{a}$ & $25-31$ & $11-14$ & $6-9$ & $3-5$ & $3-3$ & $0-0$ \\
\hline Croatia $^{a}$ & 28-34 & $10-13$ & $6-9$ & $3-5$ & $3-3$ & $0-0$ \\
\hline Cyprus $^{a}$ & $31-38$ & $9-12$ & $1-2$ & $1-2$ & $2-2$ & $1-1$ \\
\hline Czech Republic $^{a}$ & $24-29$ & $11-15$ & $6-9$ & $3-5$ & $3-3$ & $0-0$ \\
\hline Denmark $^{a}$ & $50-60$ & $4-5$ & $1-2$ & $1-2$ & $2-2$ & $1-1$ \\
\hline Estonia $^{a}$ & $28-33$ & $10-13$ & $6-9$ & $3-5$ & $3-3$ & $0-0$ \\
\hline Finland $^{\mathrm{a}}$ & $32-38$ & $11-14$ & $0-0$ & $0-0$ & $0-1$ & $0-0$ \\
\hline France & $57-75$ & $3-9$ & $0.6-1.8$ & 5.6-NA & $3.5-\mathrm{NA}$ & $1-\mathrm{NA}$ \\
\hline Germany & 45-NA & 10-NA & $2-N A$ & $1-1$ & $1-2$ & $0-0$ \\
\hline Greece & 22-NA & $12-N A$ & 2.3-NA & $7-10$ & $4-5$ & $0-0$ \\
\hline Hungary ${ }^{a}$ & $27-33$ & $10-13$ & $6-9$ & $3-5$ & $3-3$ & $0-0$ \\
\hline Iceland $^{a}$ & $36-43$ & $10-12$ & $0-0$ & $0-0$ & $0-0$ & $0-0$ \\
\hline Ireland & $10-N A$ & 3-NA & $0.9-\mathrm{NA}$ & $0.1-\mathrm{NA}$ & $0.5-\mathrm{NA}$ & $0.4-N A$ \\
\hline Italy & 37-NA & 19-NA & 5.2-NA & 2.5-NA & 2.7-NA & $0.7-\mathrm{NA}$ \\
\hline Latvia $^{a}$ & $29-35$ & $10-13$ & $6-9$ & $3-5$ & $3-3$ & $0-0$ \\
\hline Lithuania $^{a}$ & $29-36$ & $10-13$ & $6-9$ & $3-5$ & $3-3$ & $0-0$ \\
\hline Luxembourg $^{a}$ & $49-59$ & $4-5$ & $1-1$ & $1-1$ & $1-1$ & $0-1$ \\
\hline Malta $^{a}$ & $56-68$ & $2-3$ & $1-2$ & $1-2$ & $2-2$ & $1-1$ \\
\hline Netherlands & 15-19 & $2-2$ & $0.2-0.3$ & $0.1-0.2$ & $0.6-0.6$ & $0.2-0.3$ \\
\hline Norway $^{a}$ & $41-50$ & $7-8$ & $1-2$ & $1-2$ & $2-2$ & $1-1$ \\
\hline Poland ${ }^{a}$ & $28-34$ & $9-12$ & $8-11$ & $4-6$ & $3-3$ & $0-0$ \\
\hline Portugal $^{a}$ & 39-48 & $7-9$ & $1-2$ & $1-2$ & $2-2$ & $1-1$ \\
\hline Romania $^{a}$ & $22-27$ & $12-15$ & $6-9$ & $3-5$ & $3-3$ & $0-0$ \\
\hline Slovakia ${ }^{a}$ & 29-35 & $10-13$ & $4-6$ & $3-4$ & $3-3$ & $0-0$ \\
\hline Slovenia $^{\mathrm{a}}$ & $36-44$ & $8-11$ & $1-2$ & $1-2$ & $2-2$ & $1-1$ \\
\hline Spain & $23-51$ & $5-12$ & $0.9-3.1$ & $0.9-\mathrm{NA}$ & $2.1-3.1$ & $1-1.6$ \\
\hline Sweden & $9-30$ & $6-N A$ & 1.2-NA & $1-2$ & $2-2$ & $1-1$ \\
\hline Switzerland ${ }^{a}$ & $23-28$ & $14-18$ & $0-1$ & $0-0$ & $1-1$ & $0-0$ \\
\hline UK & $39-54$ & $8-16$ & $1.5-3.1$ & $2.7-5.5$ & $2.7-5.7$ & $0.6-1$ \\
\hline
\end{tabular}

${ }^{a}$ countries where primary care incidence was predicted

older age groups. This pattern tends to occur in countries in Eastern and Southern Europe, as has also been observed in a previous review [3].

According to our estimates, most varicella cases (54\%) lead to a physician consultation and a small proportion of cases $(0.3 \%)$ are hospitalised. We found that the highest consultation rates $(100 \%)$ occurred among children aged 10 to 14 years, while the highest hospitalisation rates $(1.3 \%)$ were in 20 to 39 year olds. Case fatality rate was highest $(0.03 \%)$ in the $>40$ years age group followed by the 20 to 39 years age group $(0.005 \%)$. These findings confirm that the majority of disease burden is in the younger age groups, but disease is more severe in adults and the elderly [4].

The main strength of our study is that we followed a systematic approach to quantify age-specific varicella incidence. In this way, we maximised transparency and comparability across countries. We based our estimates on the best available evidence, as obtained through a comprehensive SLR of the epidemiology of varicella. To estimate varicella incidence at community level we used seroprevalence data. Unlike other surveillance data, seroprevalence data are not affected by health care seeking or under-reporting and therefore provide a more accurate 
Table 6 Age-specific annual mortality incidence $(/ 100,000)$ of varicella in European countries before the introduction of universal childhood immunization programs

\begin{tabular}{|c|c|c|c|c|c|c|}
\hline \multirow[t]{2}{*}{ Country } & \multicolumn{6}{|c|}{ Annual incidence $/ 100,000(95 \% \mathrm{Cl})$ per age group in years } \\
\hline & $<5$ & $5-9$ & $10-14$ & $15-19$ & $20-39$ & $40+$ \\
\hline Austria & $0.08(0-1.06)$ & $0(0-0.91)$ & $0(0-0.89)$ & $0(0-0.81)$ & $0(0-0.17)$ & $0(0-0.09)$ \\
\hline Belgium & $0.13(0-0.81)$ & $0.02(0-0.59)$ & $0.02(0-0.63)$ & $0(0-0.58)$ & $0(0-0.13)$ & $0.02(0-0.11)$ \\
\hline Bulgaria & $0.03(0-1.16)$ & $0(0-1.08)$ & $0(0-1.16)$ & $0(0-1.17)$ & $0(0-0.2)$ & $0(0-0.09)$ \\
\hline Croatia & $0.09(0-1.97)$ & $0.04(0-1.82)$ & $0(0-1.79)$ & $0(0-1.52)$ & $0.01(0-0.35)$ & $0(0-0.16)$ \\
\hline Cyprus & $0(0-7.45)$ & $0(0-7.77)$ & $0(0-8.15)$ & $0(0-7)$ & $0(0-1.32)$ & $0(0-0.93)$ \\
\hline Czech Rep. & $0(0-0.66)$ & $0.02(0-0.68)$ & $0(0-0.78)$ & $0(0-0.8)$ & $0(0-0.12)$ & $0(0-0.07)$ \\
\hline Denmark & $0.06(0-1.36)$ & $0(0-1.11)$ & $0(0-1.11)$ & $0(0-1.05)$ & $0(0-0.26)$ & $0(0-0.13)$ \\
\hline Estonia & $0(0-5.11)$ & $0(0-4.9)$ & $0(0-5.84)$ & $0(0-6.17)$ & $0(0-1.03)$ & $0(0-0.54)$ \\
\hline Finland & $0(0-1.22)$ & $0(0-1.21)$ & $0(0-1.26)$ & $0(0-1.2)$ & $0(0-0.27)$ & $0.03(0-0.19)$ \\
\hline France & $0.07(0.01-0.22)$ & $0.02(0-0.13)$ & $0.01(0-0.11)$ & $0(0-0.1)$ & $0.01(0-0.04)$ & $0.02(0.01-0.05)$ \\
\hline Germany & $0.03(0-0.17)$ & $0.01(0-0.13)$ & $0.01(0-0.12)$ & $0(0-0.09)$ & $0(0-0.03)$ & $0(0-0.02)$ \\
\hline Greece & $0(0-0.73)$ & $0.02(0-0.71)$ & $0.02(0-0.73)$ & $0(0-0.68)$ & $0.03(0-0.18)$ & $0(0-0.06)$ \\
\hline Hungary & $0.06(0-0.95)$ & $0.02(0-0.79)$ & $0(0-0.77)$ & $0(0-0.71)$ & $0.01(0-0.15)$ & $0(0-0.08)$ \\
\hline Iceland & $0(0-16.04)$ & $0(0-16)$ & $0(0-17.3)$ & $0(0-16.71)$ & $0(0-3.97)$ & $0(0-2.52)$ \\
\hline Ireland & $0(0-1.04)$ & $0.05(0-1.17)$ & $0(0-1.19)$ & $0(0-1.26)$ & $0(0-0.29)$ & $0.06(0-0.3)$ \\
\hline Italy & $0.04(0-0.2)$ & $0.01(0-0.14)$ & $0.01(0-0.15)$ & $0(0-0.14)$ & $0.01(0-0.04)$ & $0.01(0-0.02)$ \\
\hline Latvia & $0(0-3.78)$ & $0(0-3.48)$ & $0(0-3.97)$ & $0(0-4.15)$ & $0(0-0.68)$ & $0(0-0.35)$ \\
\hline Lithuania & $0(0-2.47)$ & $0(0-2.67)$ & $0.06(0-2.82)$ & $0(0-2.15)$ & $0(0-0.49)$ & $0(0-0.24)$ \\
\hline Luxembourg & $0(0-11.45)$ & $0(0-11.66)$ & $0(0-11.59)$ & $0(0-10.99)$ & $0(0-2.3)$ & $0(0-1.35)$ \\
\hline Malta & $0(0-17.66)$ & $0(0-18.37)$ & $0(0-18.16)$ & $0(0-15.28)$ & $0(0-3.02)$ & $0(0-1.69)$ \\
\hline Netherlands & $0.04(0-0.5)$ & $0.01(0-0.42)$ & $0.01(0-0.39)$ & $0(0-0.37)$ & $0(0-0.09)$ & $0.02(0-0.08)$ \\
\hline Norway & $0(0-1.18)$ & $0(0-1.16)$ & $0(0-1.2)$ & $0(0-1.13)$ & $0.01(0-0.28)$ & $0.07(0.01-0.27)$ \\
\hline Poland & $0.03(0-0.25)$ & $0.03(0-0.23)$ & $0(0-0.2)$ & $0(0-0.19)$ & $0(0-0.04)$ & $0(0-0.03)$ \\
\hline Portugal & $0.03(0-0.88)$ & $0.03(0-0.8)$ & $0(0-0.69)$ & $0(0-0.67)$ & $0.02(0-0.18)$ & $0.01(0-0.08)$ \\
\hline Romania & $0.09(0-0.56)$ & $0(0-0.34)$ & $0.01(0-0.36)$ & $0(0-0.34)$ & $0(0-0.07)$ & $0(0-0.04)$ \\
\hline Slovakia & $0(0-1.31)$ & $0.04(0-1.39)$ & $0(0-1.4)$ & $0(0-1.26)$ & $0(0-0.22)$ & $0(0-0.14)$ \\
\hline Slovenia & $0(0-3.37)$ & $0(0-3.56)$ & $0(0-4.02)$ & $0(0-3.88)$ & $0(0-0.67)$ & $0.02(0-0.36)$ \\
\hline Spain & $0.06(0-0.27)$ & $0.02(0-0.18)$ & $0.01(0-0.19)$ & $0(0-0.18)$ & $0.03(0.01-0.08)$ & $0.01(0-0.04)$ \\
\hline Sweden & $0.12(0-0.86)$ & $0.02(0-0.69)$ & $0.04(0-0.79)$ & $0(0-0.7)$ & $0(0-0.15)$ & $0.04(0-0.14)$ \\
\hline Switzerland & $0.03(0-0.94)$ & $0.03(0-0.96)$ & $0(0-0.92)$ & $0(0-0.84)$ & $0(0-0.17)$ & $0.03(0-0.14)$ \\
\hline UK & $0.11(0.03-0.27)$ & $0.03(0-0.16)$ & $0.01(0-0.12)$ & $0.01(0-0.12)$ & $0.02(0-0.05)$ & $0.05(0.03-0.08)$ \\
\hline
\end{tabular}

Table 7 Norway: varicella age-specific annual community incidence $(/ 100,000)$ before the introduction of universal childhood immunization programs, predicted vs observed

\begin{tabular}{lll}
\hline Age group (years) & $\begin{array}{l}\text { Predicted incidence } \\
\text { per 100,000 (95\% Cl) }\end{array}$ & $\begin{array}{l}\text { Observed incidence } \\
\text { per 100,000 (95\% Cl) }\end{array}$ \\
\hline$<5$ & $13,279(10,433-16,124)$ & $9164(8100-10,344)$ \\
$5-9$ & $5372(4653-6092)$ & $8174(6526-9644)$ \\
$10-14$ & $272(0-916)$ & $240(70-486)$ \\
$15-19$ & $213(0-698)$ & $218(68-414)$ \\
$20-39$ & $123(0-372)$ & $174(62-287)$ \\
$\geq 40$ & $48(0-113)$ & $114(53-149)$ \\
\hline
\end{tabular}

representation of the incidence at the community level, although using seroprevalence data requires the assumption of time homogeneity (Bollaerts K, Riera-Montes M, Hens $\mathrm{N}$ et al. A systematic review of varicella seroprevalence in European countries before universal childhood immunization: deriving incidence from seroprevalence data. Submitted 2017). Seroprevalence data are robust and have previously been used to estimate varicella incidence in Luxembourg, Italy and Spain [10, 40, 129].

Our study has several limitations. Data sources providing PCI, HI and mortality may be affected by underascertainment and underreporting, and we may therefore 
Table 8 Norway: Varicella age-specific annual primary care incidence $(/ 100,000)$ before the introduction of universal childhood immunization programs, predicted versus observed

\begin{tabular}{|c|c|c|c|}
\hline \multirow{2}{*}{$\begin{array}{l}\text { Age group } \\
\text { (years) }\end{array}$} & \multirow{2}{*}{$\begin{array}{l}\text { Observed } \\
\text { incidence } \\
\text { per } 100,000\end{array}$} & \multicolumn{2}{|c|}{ Predicted incidence per 100,000} \\
\hline & & $\begin{array}{l}\text { Extrapolation from } \\
\text { observed community } \\
\text { incidence }\end{array}$ & $\begin{array}{l}\text { Extrapolation from } \\
\text { predicted community } \\
\text { incidence }\end{array}$ \\
\hline$<5$ & 2030 & $4851-5410$ & $7053-7865$ \\
\hline $5-9$ & 919 & $3517-4927$ & $2311-3238$ \\
\hline $10-14$ & 189 & $305-655$ & $346-742$ \\
\hline $15-19$ & 104 & $144-311$ & $141-304$ \\
\hline 20-39 & 84 & $121-223$ & $86-158$ \\
\hline$\geq 40$ & 14 & $72-117$ & $30-49$ \\
\hline
\end{tabular}

have underestimated the number of primary care visits, hospitalisations and deaths. Some of the studies we used in our estimations were regional only and might not be representative for the respective whole country. Despite our attempts to be comprehensive we cannot guarantee that all relevant data sources were identified in this review. However, we expect the number of missed data sources to be low. We did not find data for all outcomes for all countries, so we extrapolated the incidence for these countries based on data from other countries. This may have resulted in over- or underestimation of the incidence for some countries. We addressed this uncertainty by estimating 95\% CIs (for the community incidence in the $<5$ and 5-9 year olds), or by providing a minimum-maximum range otherwise. We did not consider immigration in our estimates. A seroprevalence study carried out in adults in the Netherlands [64] showed that immigrants, in particular first generation immigrants, were more likely to be varicella seronegative compared to Dutch-born adults. Studies in the UK, Ireland and Spain among pregnant women have also shown that foreign-born women are more likely to be susceptible to varicella [36, 44, 47, 57]. There is a lack of studies addressing the impact of immigration on varicella epidemiology. Most seroprevalence surveys are carried out using residual serum samples with no information on immigration status [41]. As evidenced by van Rijckevorsel et al. [64], varicella serological profiles may show geographical differences within countries, with urban areas (being areas where immigration is typically concentrated) often presenting a higher proportion of varicella susceptible adults. To estimate varicella mortality, we used data from the WHO DMDB [11]. Mortality causes are coded using International Classification of Diseases (ICD)-9 or ICD-10 and it is difficult to ascertain the accuracy of the coding. Hence misclassification of the cause of death cannot be excluded. This may have resulted in over or underestimation of varicella mortality.

Given the limited number of studies that looked at specific complications and/or sequelae, we did not model these outcomes separately. It is however noteworthy that in addition to the immediate burden on the health care system as discussed in this paper, varicella may also cause complications and long term sequelae. In children (0-17 years), reported incidence of varicella complications requiring hospitalisation ranges from 0.82 per 100,000 of the population in the UK and Ireland [119], to 19 per 100,000 in Belgium [75]. Differences in incidence are probably related to differences in the definitions used for complicated varicella. In the UK study [119] only severe complications were included, excluding secondary skin infections, while the Belgian study [75] included any complication. The most frequent complications reported were bacterial superinfections, followed by varicella pneumonia and neurological complications. Concerning long term sequelae, these are usually a result of neurological complications of varicella. Long term sequelae have been reported in 0.4 to $8 \%$ of children hospitalised for varicella $[85,95,100,103,130]$.

Recent published data from Norway provided us with an opportunity to validate the methodology used. We found that our model overestimated the varicella community incidence in Norway in $<5$ year olds and underestimated the incidence in 5 to 9 year olds. There were small differences in the predicted vs. observed incidence

Table 9 Annual number of varicella cases, consultations, hospitalizations and deaths and consultation, hospitalization and case fatality rates in Europe before the introduction of universal childhood immunization programs

\begin{tabular}{|c|c|c|c|c|c|c|c|}
\hline \multirow[t]{2}{*}{ Outcome } & \multicolumn{7}{|c|}{ Age group (years) } \\
\hline & $<5$ & $5-9$ & $10-14$ & $15-19$ & 20-39 & $40+$ & Total \\
\hline $\mathrm{N}$ varicella cases & $3,029,226$ & $1,816,442$ & 175,020 & 116,926 & 244,923 & 141,087 & $5,523,624$ \\
\hline $\mathrm{N}$ varicella consultations & $1,660,087$ & 826,610 & 179,400 & 67,609 & 164,118 & 78,015 & $2,975,839$ \\
\hline $\mathrm{N}$ varicella hospitalizations & 9905 & 2386 & 712 & 718 & 3209 & 1233 & 18,163 \\
\hline $\mathrm{N}$ varicella deaths & 16 & 5 & 2 & 1 & 12 & 45 & 81 \\
\hline Proportion of varicella cases consulting a physician (\%) & 54.80 & 45.51 & 100.00 & 57.82 & 67.01 & 55.30 & 53.87 \\
\hline Proportion of varicella cases that are hospitalized (\%) & 0.33 & 0.13 & 0.41 & 0.61 & 1.31 & 0.87 & 0.33 \\
\hline Case fatality rate (\%) & 0.001 & 0.000 & 0.001 & 0.001 & 0.005 & 0.032 & 0.001 \\
\hline
\end{tabular}




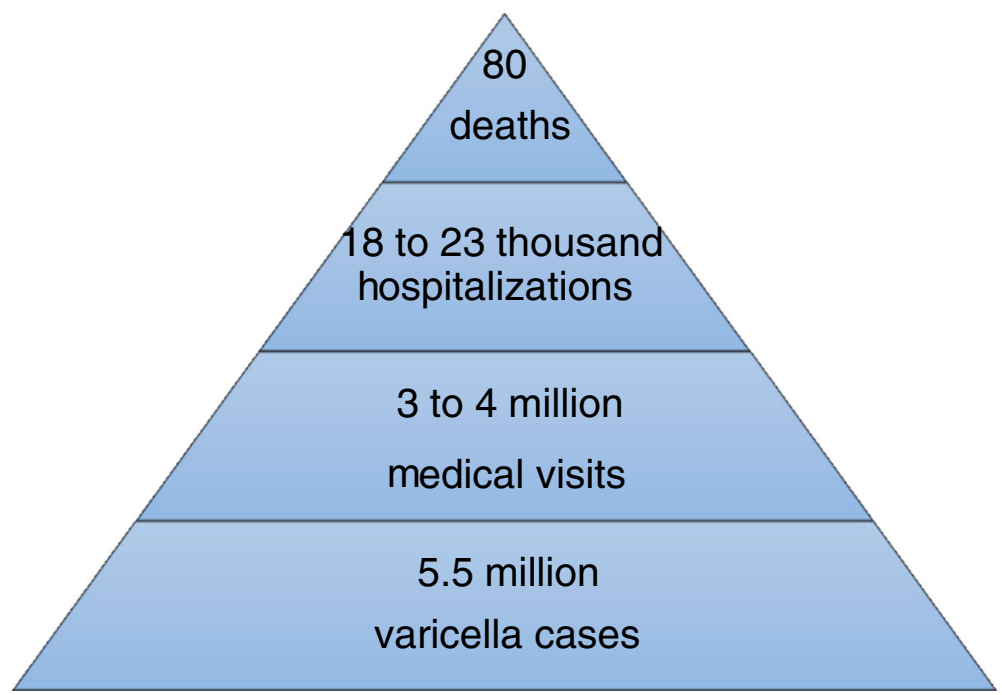

Fig. 2 Annual burden of varicella in Europe

Table 10 Number of estimated varicella cases occurring in European countries every year, number of varicella cases reported to EUVAC

\begin{tabular}{|c|c|c|c|c|c|}
\hline Country & $\begin{array}{l}\text { Annual estimated number } \\
\text { of cases in the community } \\
\text { (All ages) }\end{array}$ & $\begin{array}{l}\text { Number of varicella } \\
\text { cases reported to } \\
\text { EUVAC.NET (2009) }\end{array}$ & $\begin{array}{l}\text { Number of varicella } \\
\text { cases reported to } \\
\text { EUVAC.NET (2010) }\end{array}$ & $\begin{array}{l}\text { Average number of } \\
\text { cases reported to } \\
\text { EUVAC.NET (2009/10) }\end{array}$ & $\begin{array}{l}\text { Reported cases/estimated } \\
\text { total cases (\%) }\end{array}$ \\
\hline Bulgaria & 68,840 & 29,117 & 19,724 & 24,421 & 35 \\
\hline Croatia & 43,380 & 17,563 & 16,027 & 16,795 & 39 \\
\hline Cyprus & 9387 & 159 & 75 & 117 & 1 \\
\hline Czech Republic & 112,559 & 47,192 & 48,270 & 47,731 & 42 \\
\hline Estonia & 14,846 & 8556 & 6146 & 7351 & 50 \\
\hline Finland $^{a}$ & 58,715 & 360 & 358 & 359 & 1 \\
\hline Greece $^{b}$ & 109,214 & 7 & 5 & 6 & 0 \\
\hline Hungary & 97,591 & 40,460 & 39,602 & 40,031 & 41 \\
\hline Italy & 597,881 & 56,502 & 39,649 & 48,076 & 8 \\
\hline Latvia & 20,772 & 5019 & 3697 & 4358 & 21 \\
\hline Lithuania & 28,498 & 12,698 & 11,042 & 11,870 & 42 \\
\hline Malta & 4524 & 183 & 92 & 138 & 3 \\
\hline Norway ${ }^{c}$ & 63,105 & 31 & NR & 31 & 0 \\
\hline Poland & 403,362 & 140,115 & 183,446 & 161,781 & 40 \\
\hline Romania & 206,872 & 44,693 & 36,245 & 40,469 & 20 \\
\hline Slovakia & 57,449 & 17,735 & 19,887 & 18,811 & 33 \\
\hline Slovenia & 21,729 & 13,060 & 9087 & 11,074 & 51 \\
\hline Spain & 478,816 & 141,399 & 157,222 & 149,311 & 31 \\
\hline
\end{tabular}

NR not reported

${ }^{a}$ Finland has a laboratory based surveillance system which does not separate clinical disease and therefore includes both varicella and herpes zoster. Only laboratory confimed cases are reported. ${ }^{\mathrm{b}}$ Greece: the national mandatory surveillance system includes only varicella cases with complications. ${ }^{\mathrm{C}}$ Norway: only laboratory confirmed cases of varicella encephalitis reported.

NET during 2009 and 2010, and percentage of reported cases out of the total number of estimated varicella cases occurring in the community 
for ages 10 to 39 , with the model underestimating the incidence in the $\geq 40$ year olds. The model we used to estimate community incidence was based on the prediction variables including the percentage of children $<3$ years of age receiving formal childcare and population density. While for both prediction variables Norway is similar to northern European countries with the highest incidence observed in children $<5$ years old, the varicella epidemiology pattern in Norway also somewhat resembles that of southern European countries with a relatively high observed incidence in the 5 to 9 year olds. Although we explored the inclusion of additional explanatory variables in our model, none of them improved the fit of the model. The methodology we used to estimate PCI overestimated the observed PCI in Norway, particularly in the younger age groups. We used the minimum-maximum observed PCR to estimate PCI in countries without data. Health care seeking behaviour, and hence the PCR, varies strongly between countries [104]. For example, $38 \%$ of children $<5$ years with varicella consulting a physician [131] in the Netherlands compared to $88 \%$ of children $<3$ years in France [132]. There may also be differences in hospitalisation policies for varicella cases between countries which potentially affect the hospitalisation rate. We tried to address this uncertainty by estimating a minimum-maximum range for PCI and HI. However, we cannot exclude that PCI and $\mathrm{HI}$ may have been over or underestimated for some countries, like in the case of Norway.

\section{Conclusions}

In conclusion, the estimated burden of varicella in Europe in the pre-immunization period was substantial with more than 5 million new cases estimated annually, of which slightly more than half is expected to lead to a physician consultation, about 20,000 to hospitalisation, and up to 80 to death. Since very few countries or regions have introduced universal childhood varicella immunization programs, these figures are probably still true today. Although the main share of the burden is in children $<5$ years old, adults require hospitalisation more often and are at higher risk of death. This information should be considered when planning and evaluating varicella control strategies. Improving and standardizing varicella surveillance in Europe, as initiated by ECDC, will be important to improve the quality of data available and allow better inter-country comparison. There is also a need to better understand the driving factors of country-specific differences in varicella transmission and health care utilization. In addition, future research on sero-epidemiology with prospective sampling and data collection, ensuring the inclusion of migrant populations, would further improve our understanding of the epidemiology of varicella in Europe.

\section{Additional files}

Additional file 1: Risk of bias assessment tool. (DOCX $15 \mathrm{~kb})$
Additional file 2: Variables used in the linear regression models. (DOCX $13 \mathrm{~kb}$ )
Additional file 3: Study characteristics. (XLSX $12 \mathrm{~kb})$
Additional file 4: Data extraction tables. (XLSX $308 \mathrm{~kb})$
Additional file 5: Country and age-specific number of varicella cases in
the community, primary care visits, hospitalizations and deaths. (XLSX $24 \mathrm{~kb}$ )

\section{Abbreviations}

Cl: Confidence interval; DMDB: Detailed Mortality Database; ECDC: European Centre for Disease Prevention and Control; EMA: European Medicines Agency; EU: European Union; HI: Hospitalisation incidence; HR: Hospitalisation rate; PCl: Primary care incidence; PCR: Primary care rate; SDC: Supplementary Digital Content; SLR: Systematic literature review; WHO: World Health Organisation

\section{Acknowledgements}

The authors would like to thank Martine Sabbe (WIV-ISP, Belgium), Jan Kynd (SZU, Czech Republic), Daniel Levy-Bruhl (InVS, France), Thorolfur Gudnason (Landlaeknir, Iceland), Suzanne Cotter (HSE, Ireland), Joke Bilcke (Antwerp University), Alies van Lier (RIVM), Hester de Melker (RIVM), Maria Cristina Rota (Istituto Superiore di Sanità Rome, Italy) and Paloma Carrillo-Santisteve (ECDC) for providing data sources and/or references. The authors also thank Marc Baay (P95) for support with the systematic literature review and Sally Jackson (P95) for writing services.

Funding

This work is funded by Sanofi Pasteur, MSD, France.

\section{Availability of data and materials}

All data generated or analysed during this study are included in this published article (and its Additional files 1, 2, 3, 4 and 5).

\section{Authors' contributions}

The idea and contents of the article emerged from discussions among $\mathrm{SH}$, $M R, K B$ and TV, who have experience in epidemiology and biostatistics. SH, $\mathrm{MR}, \mathrm{KB}$ and TV designed the study. AS, UH and $\mathrm{NH}$ provided input to study design. MR conducted the systematic review. KB implemented the statistical analyses. $\mathrm{UH}, \mathrm{NH}, \mathrm{GG}, \mathrm{AG}, \mathrm{BN}, \mathrm{GM}$ and $\mathrm{EF}$ provided input in the interpretation of the results. MR wrote the first manuscript draft. All authors critically reviewed the subsequent revisions and approved the final version. MR is the guarantor.

\section{Competing interests}

$\mathrm{KB}, \mathrm{MR}$ and TV received consulting fees from SPMSD for this work. SH is a current SPMSD employee, AS is mandated by SPMSD. No honoraria were paid to $U H, N H, G G, A G, B N, G M$, and $E F$ for this work. $U H, N H, G M$ and $E F$ have no conflicts to declare. GG received grants from GSK Biologicals SA, SPMSD, Novartis, Crucell/Janssen, Sequirus and Pfizer as consultant, advisory board member, expert, speaker, organizer of congresses/conferences or as investigator in clinical trials. BN received professional fees from SP for attending advisory panels and other consultancy projects. NH gratefully acknowledges support from the University of Antwerp scientific chair in Evidence-Based Vaccinology, financed in 2009-2016 by a gift from Pfizer and in 2016 by GSK

\section{Consent for publication}

Not applicable.

Ethics approval and consent to participate Not applicable.

\section{Publisher's Note}

Springer Nature remains neutral with regard to jurisdictional claims in published maps and institutional affiliations.

\section{Author details}

${ }^{1}$ P95 Pharmacovigilance and Epidemiology Services, Koning Leopold III Laan 1, 3001 Leuven, Belgium. ${ }^{2}$ Division of Paediatric Infectious Diseases and 
Vaccinology, University of Basel Children's Hospital, CH-4056 Basel, Switzerland. Interuniversity Institute for Biostatistics and statistical Bioinformatics, Hasselt University, Antwerp, Belgium. ${ }^{4}$ Centre for Health Economics Research and Modelling Infectious Diseases and Centre for the Evaluation of Vaccination, Vaccine \& Infectious Disease Institute, University of Antwerp, Antwerp, Belgium. ${ }^{5}$ Department of Medical Sciences, University of Ferrara, Ferrara, Italy. ${ }^{6}$ Universidad Rey Juan Carlos, Madrid, Spain. ${ }^{7}$ Department of Primary Care and Public Health, Imperial College London, London, UK. ${ }^{8}$ Department of Infectious Disease Epidemiology and Modelling, Norwegian Institute of Public Health, Oslo, Norway. ${ }^{9}$ Aixial, 4 rue Danjou, 92513 Boulogne-Billancourt, France. ${ }^{10}$ Sanofi Pasteur MSD, 162 avenue Jean Jaurès, 69007 Lyon, France.

\section{Received: 26 October 2016 Accepted: 7 May 2017}

Published online: 18 May 2017

\section{References}

1. Arvin AM. Varicella-zoster virus. Clin Microbiol Rev. 1996;9:361-81.

2. Bonanni P, Breuer J, Gershon A, et al. Varicella vaccination in Europe - taking the practical approach. BMC Med. 2009;7:26.

3. Helmuth IG, Poulsen A, Suppli CH, Molbak K. Varicella in Europe-a review of the epidemiology and experience with vaccination. Vaccine. 2015;33:2406-13.

4. European Centre for Disease Prevention and Control (ECDC). ECDC guidance: Varicella vaccination in the European Union, 2015. Available from: http://ecdc europa.eu/en/publications/Publications/Varicella-Guidance-2015.pdf.

5. EUVAC.NET. Surveillance of Varicella and Herpes Zoster in Europe, November 2010. Available from: http://ecdc.europa.eu/en/publications/Publications/ varicella_zoster_report_2009_euvacnet.pdf.

6. Bonhoeffer J, Baer G, Muehleisen B, et al. Prospective surveillance of hospitalisations associated with varicella-zoster virus infections in children and adolescents. Eur J Pediatr. 2005;164:366-70.

7. Socan M. Evaluation of mandatory case-based reporting system for varicella in the prevaccine era. Cent Eur J Public Health. 2010;18:99-103.

8. Fleming DM, Schellevis FG, Falcao I, Alonso TV, Padilla ML. The incidence of chickenpox in the community. Lessons for disease surveillance in sentinel practice networks. Eur J Epidemiol. 2001;17:1023-7.

9. Hoy D, Brooks P, Woolf A, et al. Assessing risk of bias in prevalence studies: modification of an existing tool and evidence of interrater agreement. J Clin Epidemiol. 2012;65:934-9.

10. Ciofi Degli Atti ML, Rota MC, Mandolini D, et al. Assessment of varicella underreporting in Italy. Epidemiol Infect. 2002;128:479-84.

11. WHO European Detailed Mortality Database. WHO Regional Office for Europe. Available at: http://data.euro.who.int/dmdb/.

12. Santermans E, Goeyvaerts N, Melegaro A, et al. The social contact hypothesis under the assumption of endemic equilibrium: elucidating the transmission potential of VZV in Europe. Epidemics. 2015;11:14-23.

13. Silhol R, Alvarez FP, Arena $C$, et al. Micro and macro population effects in disease transmission: the case of varicella. Epidemiol Infect. 2010;138:482-90.

14. European Commission. Eurostat database. Available at: http://ec.europa.eu/ eurostat/data/database.

15. WHO Global Health Expenditure Database. Available at http://apps.who.int/ nha/database/Home/Index/en.

16. Rimseliene G, Vainio K, Gibory M, Salamanca BV, Flem E. Varicella-Zoster virus susceptibility and primary healthcare consultations in Norway. BMC Infect Dis. 2016:16:254

17. Instituto de Salud Carlos III. Centro Nacional de Epidemiología. Estudio seroepidemiológico: situación de las enfermedades vacunables en España. Available from: http://www.isciii.es/ISCIII/es/contenidos/fd-servicios-cientificotecnicos/fd-vigilancias-alertas/fd-enfermedades/SEROEPIDEMIOLOGICO.pdf.

18. Aebi C, Fischer K, Gorgievski M, Matter L, Muhlemann K. Age-specific seroprevalence to varicella-zoster virus: study in Swiss children and analysis of European data. Vaccine. 2001;19:3097-103.

19. Alanen A, Kahala K, Vahlberg T, Koskela P, Vainionpaa R. Seroprevalence, incidence of prenatal infections and reliability of maternal history of varicella zoster virus, cytomegalovirus, herpes simplex virus and parvovirus B19 infection in south-western Finland. BJOG. 2005:112:50-6.

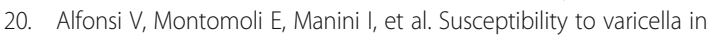
childbearing age women, Central Italy: is there a need for vaccinating this population group? Vaccine. 2007;25:6086-8.
21. Baer G, Bonhoeffer J, Schaad UB, Heininger U. Seroprevalence and immunization history of selected vaccine preventable diseases in medical students. Vaccine. 2005;23:2016-20.

22. De Juanes JR, Gil A, San-Martin M, Gonzalez A, Esteban J, Garcia de Codes A Seroprevalence of varicella antibodies in healthcare workers and health sciences students. Reliability of self-reported history of varicella. Vaccine. 2005:23:1434-6.

23. de Melker $H$, Berbers $G$, Hahne $S$, et al. The epidemiology of varicella and herpes zoster in The Netherlands: implications for varicella zoster virus vaccination. Vaccine. 2006:24:3946-52.

24. Diez-Domingo J, Gil A, San-Martin M, et al. Seroprevalence of varicella among children and adolescents in Valencia, Spain. Reliability of the parent's reported history and the medical file for identification of potential candidates for vaccination. Hum Vaccin. 2005:1:204-6.

25. Fedeli U, Zanetti C, Saia B. Susceptibility of healthcare workers to measles, mumps rubella and varicella. J Hosp Infect. 2002;51:133-5.

26. Gabutti G, Penna C, Rossi M, et al. The seroepidemiology of varicella in Italy. Epidemiol Infect. 2001;126:433-40.

27. Gabutti G, Rota MC, Guido M, et al. The epidemiology of Varicella zoster virus infection in Italy. BMC Public Health. 2008:8:372.

28. Gallagher J, Quaid B, Cryan B. Susceptibility to varicella zoster virus infection in health care workers. Occup Med (Lond). 1996:46:289-92.

29. Gil A, Gonzalez A, Dal-Re R, Ortega P, Dominguez V. Prevalence of antibodies against varicella zoster, herpes simplex (types 1 and 2), hepatitis $B$ and hepatitis a viruses among Spanish adolescents. J Inf Secur. 1998;36:53-6.

30. Guido M, Tinelli A, De Donno A, et al. Susceptibility to varicella-zoster among pregnant women in the province of Lecce, Italy. J Clin Virol. 2012;53:72-6.

31. Heininger U, Baer G, Bonhoeffer J, Schaad UB. Reliability of varicella history in children and adolescents. Swiss Med Wkly. 2005;135:252-5.

32. Heininger U, Braun-Fahrlander C, Desgrandchamps D, et al. Seroprevalence of varicella-zoster virus immunoglobulin $\mathrm{G}$ antibodies in Swiss adolescents and risk factor analysis for seronegativity. Pediatr Infect Dis J. 2001:20:775-8

33. Heininger $U$, Desgrandchamps D, Schaad UB. Seroprevalence of Varicellazoster virus IgG antibodies in Swiss children during the first 16 months of age. Vaccine. 2006;24:3258-60.

34. Kavaliotis J, Petridou S, Karabaxoglou D. How reliable is the history of chickenpox? Varicella serology among children up to 14 years of age. Int J Infect Dis. 2003:7:274-7.

35. Khoshnood B, Debruyne M, Lancon F, et al. Seroprevalence of varicella in the French population. Pediatr Infect Dis J. 2006:25:41-4

36. Knowles SJ, Grundy K, Cahill I, Cafferkey MT. Susceptibility to infectious rash illness in pregnant women from diverse geographical regions. Commun Dis Public Health. 2004;7:344-8.

37. Koskiniemi M, Lappalainen M, Schmid DS, Rubtcova E, Loparev VN. Genotypic analysis of varicella-zoster virus and its seroprevalence in Finland. Clin Vaccine Immunol. 2007;14:1057-61.

38. Kudesia G, Partridge S, Farrington CP, Soltanpoor N. Changes in age related seroprevalence of antibody to varicella zoster virus: impact on vaccine strategy. J Clin Pathol. 2002:55:154-5.

39. Leuridan E, Hens N, Hutse V, Aerts M, Van Damme P. Kinetics of maternal antibodies against rubella and varicella in infants. Vaccine. 2011;29:2222-6.

40. Mossong J, Putz L, Schneider F. Seroprevalence and force of infection of varicella-zoster virus in Luxembourg. Epidemiol Infect. 2004;132:1121-7.

41. Nardone A, de Ory F, Carton M, et al. The comparative sero-epidemiology of varicella zoster virus in 11 countries in the European region. Vaccine. 2007;25:7866-72.

42. Oliveira J, da Cunha S, Corte-Real R, Sampaio L, Dais N, Melico-Silvestre A The prevalence of measles, rubella, mumps and chickenpox antibodies in a population of health care workers. Acta Medica Port. 1995;8:206-16.

43. Oliveira J, Dias N, Ferreira TM, et al. The determination of susceptibility to measles, rubella, mumps and chickenpox in students of health-related areas. Acta Medica Port. 1999:12:155-60.

44. Pembrey L, Raynor P, Griffiths P, Chaytor S, Wright J, Hall AJ. Seroprevalence of cytomegalovirus, Epstein Barr virus and varicella zoster virus among pregnant women in Bradford: a cohort study. PLoS One. 2013;8:e81881.

45. Perez-Farinos N, Garcia-Comas L, Ramirez-Fernandez R, et al. Seroprevalence of antibodies to varicella-zoster virus in Madrid (Spain) in the absence of vaccination. Cent Eur J Public Health. 2008:16:41-4

46. Pinquier D, Gagneur A, Balu L, et al. Prevalence of anti-varicella-zoster virus antibodies in French infants under 15 months of age. Clin Vaccine Immunol. 2009;16:484-7. 
47. Plans P, Costa J, Espunes J, Plasencia A, Salleras L. Prevalence of varicellazoster antibodies in pregnant women in Catalonia (Spain). Rationale for varicella vaccination of women of childbearing age. BJOG. 2007;114:1122-7.

48. Ringler M, Gobel G, Most J, Weithaler K. Fully vaccinated children are rare: immunization coverage and seroprevalence in Austrian school children. Eur J Epidemiol. 2003;18:161-70.

49. Saadatian-Elahi M, Mekki Y, Del Signore C, et al. Seroprevalence of varicella antibodies among pregnant women in Lyon-France. Eur J Epidemiol. 2007; 22:405-9.

50. Salleras $L$, Dominguez A, Plans $P$, et al. Seroprevalence of varicella zoster virus infection in child and adult population of Catalonia (Spain). Med Microbiol Immunol. 2008;197:329-33.

51. Salleras L, Dominguez A, Vidal J, Plans P, Salleras M, Taberner JL. Seroepidemiology of varicella-zoster virus infection in Catalonia (Spain). Rationale for universal vaccination programmes. Vaccine. 2000;19:183-8.

52. Sauerbrei A, Prager J, Bischoff A, Wutzler P. Antibodies against vaccinepreventable diseases in pregnant women and their offspring. Measles, mumps, rubella, poliomyelitis, and varicella. Bundesgesundheitsblatt Gesundheitsforschung Gesundheitsschutz. 2004;47:10-5.

53. Siennicka J, Trzcinska A, Rosinska M, Litwinska B. Seroprevalence of varicellazoster virus in polish population. Przegl Epidemiol. 2009:63:495-9.

54. Socan M, Berginc N, Lajovic J. Varicella susceptibility and transmission dynamics in Slovenia. BMC Public Health. 2010;10:360.

55. Suarez Gonzalez A, Otero Guerra L, De La Guerra GV, La Iglesia Martinez Pd P, Solis Sanchez G, Rodriguez Fernandez A. Varicella and parvovirus B19 immunity among pregnant women in Gijon, Spain. Med Clin (Barc). 2002;119:171-3.

56. Svahn A, Berggren J, Parke A, Storsaeter J, Thorstensson R, Linde A. Changes in seroprevalence to four herpesviruses over 30 years in Swedish children aged 9-12 years. J Clin Virol. 2006;37:118-23.

57. Talukder YS, Kafatos G, Pinot de Moira A, et al. The seroepidemiology of varicella zoster virus among pregnant Bangladeshi and white British women in the London borough of tower hamlets, UK. Epidemiol Infect. 2007;135:1344-53.

58. Thiry N, Beutels P, Shkedy Z, et al. The seroepidemiology of primary varicellazoster virus infection in Flanders (Belgium). Eur J Pediatr. 2002;161:588-93.

59. Thorarinsdottir H, Love A, Laxdal T, Gudnason T, Haraldsson A. Varicella in Icelandic children-epidemiology and complications. Laeknabladid. 2009;95:113-8.

60. Trevisan A, Frasson C, Morandin M, et al. Immunity against infectious diseases: predictive value of self-reported history of vaccination and disease. Infect Control Hosp Epidemiol. 2007;28:564-9.

61. Trevisan A, Morandin M, Frasson C, et al. Prevalence of childhood exanthematic disease antibodies in paramedical students: need of vaccination. Vaccine. 2006;24:171-6.

62. van Lier A, Smits $G$, Mollema $L$, et al. Varicella zoster virus infection occurs at a relatively young age in The Netherlands. Vaccine. 2013;31:5127-33.

63. van Rijckevorsel GG, Bovee LP, Damen M, Sonder GJ, Schim van der Loeff MF, van den Hoek A. Increased seroprevalence of IgG-class antibodies against cytomegalovirus, parvovirus B19, and varicella-zoster virus in women working in child day care. BMC Public Health. 2012;12:475.

64. van Rijckevorsel GG, Damen M, Sonder GJ, van der Loeff MF, van den Hoek A. Seroprevalence of varicella-zoster virus and predictors for seronegativity in the Amsterdam adult population. BMC Infect Dis. 2012;12:140.

65. Vandersmissen $G$, Moens $G$, Vranckx $R$, de Schryver A, Jacques $P$ Occupational risk of infection by varicella zoster virus in Belgian healthcare workers: a seroprevalence study. Occup Environ Med. 2000;57:621-6.

66. Vilibic-Cavlek T, Ljubin-Sternak S, Kolaric B, et al. Immunity to varicella-zoster virus in Croatian women of reproductive age targeted for serology testing. Arch Gynecol Obstet. 2012;286:901-4.

67. Vyse AJ, Gay NJ, Hesketh LM, Morgan-Capner P, Miller E. Seroprevalence of antibody to varicella zoster virus in England and Wales in children and young adults. Epidemiol Infect. 2004;132:1129-34.

68. Wutzler P, Farber I, Wagenpfeil S, Bisanz H, Tischer A. Seroprevalence of varicella-zoster virus in the German population. Vaccine. 2001;20:121-4.

69. HPSC Annual Epidemiological Report 2012. Available from: http://www.hpsc. ie/A-ZNaccinePreventable/VaricellaChickenpox/SurveillanceReports/ AnnualReports/.

70. HPSC Annual Epidemiological Report 2013. Available from: http://www.hpsc ie/A-ZNaccinePreventable/VaricellaChickenpox/SurveillanceReports/ AnnualReports/.

71. HPSC Annual Epidemiological Report 2014. Available from: http://www.hpsc. ie/A-ZNaccinePreventable/VaricellaChickenpox/SurveillanceReports/ AnnualReports/.
72. National Archive SDO data, Ministry of Health, General Directorate of Health and Planning, VI Office, 2001-2005.

73. Bennet R, Bogdanovic G, Giske CG, Eriksson M. More severe bacterial infections could be prevented with vaccine. Rotavirus, influenza and varicella cause thousands of hospital admissions. Lakartidningen. 2010;107:3040-3.

74. Bilcke J, Ogunjimi B, Marais C, et al. The health and economic burden of chickenpox and herpes zoster in Belgium. Epidemiol Infect. 2012;140:2096-109.

75. Blumental S, Sabbe M, Lepage P, Belgian Group for V. Varicella paediatric hospitalisations in Belgium: a 1-year national survey. Arch Dis Child. 2016; 101:16-22.

76. Boelle PY, Hanslik T. Varicella in non-immune persons: incidence, hospitalization and mortality rates. Epidemiol Infect. 2002;129:599-606.

77. Bonmarin I, Ndiaye B, Seringe E, Levy-Bruhl D. The epidemiology of varicella in France. Bull Epidemiol Hebd. 2005;8:30-2.

78. Bonsignori F, Chiappini E, Frenos S, Peraldo M, Galli L, de Martino M. Hospitalization rates for complicated and uncomplicated chickenpox in a poorly vaccined pediatric population. Infection. 2007:35:444-50.

79. Bramley JC, Jones IG. Epidemiology of chickenpox in Scotland: 1981 to 1998. Commun Dis Public Health. 2000;3:282-7.

80. Brisson M, Edmunds WJ. Epidemiology of Varicella-zoster virus in England and Wales. J Med Virol. 2003;70(Suppl 1):S9-14.

81. Brisson $M$, Edmunds WJ, Law B, et al. Epidemiology of varicella zoster virus infection in Canada and the United Kingdom. Epidemiol Infect. 2001;127:305-14.

82. Critselis E, Nastos PT, Theodoridou K, et al. Time trends in pediatric hospitalizations for varicella infection are associated with climatic changes: a 22-year retrospective study in a tertiary Greek referral center. PLoS One. 2012;7:e52016.

83. Diez Domingo J, Ridao M, Latour J, Ballester A, Morant A. A cost benefit analysis of routine varicella vaccination in Spain. Vaccine. 1999;17:1306-11.

84. Dubos F, Grandbastien B, Hue V, Martinot A. Epidemiology of hospital admissions for paediatric varicella infections: a one-year prospective survey in the pre-vaccine era. Epidemiol Infect. 2007;135:131-8.

85. Giacchino R, Losurdo G, Castagnola E. Decline in mortality with varicella vaccination. N Engl J Med. 2005;352:1819.

86. Gil A, Gonzalez A, Oyaguez I, Martin MS, Carrasco P. The burden of severe varicella in Spain, 1995-2000 period. Eur J Epidemiol. 2004;19:699-702.

87. Gil A, Oyaguez I, Carrasco P, Gonzalez A. Epidemiology of primary varicella hospitalizations in Spain. Vaccine. 2001;20:295-8.

88. Gil A, San-Martin M, Carrasco P, Gonzalez A. Epidemiology of severe varicella-zoster virus infection in Spain. Vaccine. 2004;22:3947-51.

89. Grimheden P, Bennet R, Hjern A, Nilsson A, Eriksson M. Chickenpox not always a harmless child disease. General vaccination in Sweden can prevent significant morbidity. Lakartidningen. 2009;106:580-2.

90. Guillen JM, Gil-Prieto R, Alvaro A, Gil A. Burden of adult varicella hospitalizations in Spain (2001-2007). Hum Vaccin. 2010;6:659-63.

91. Guillen JM, Samaniego-Colmenero Mde L, Hernandez-Barrera V, Gil A. Varicella paediatric hospitalizations in Spain. Epidemiol Infect. 2009;137:519-25.

92. Hervas D, Osona B, Masip C, Yeste S, Figuerola J, Hervas JA. Risk of varicella complications in children treated with inhaled steroids. Pediatr Infect Dis J. 2008;27:1113-4

93. Jelastopulu E, Merekoulias G, Alexopoulos EC. Underreporting of communicable diseases in the prefecture of Achaia, western Greece, 1999-2004 - missed opportunities for early intervention. Euro Surveill. 2010;15:19579.

94. Liese JG, Grote V, Rosenfeld E, et al. The burden of varicella complications before the introduction of routine varicella vaccination in Germany. Pediatr Infect Dis J. 2008;27:119-24.

95. Marchetto S, de Benedictis FM, de Martino M, et al. Epidemiology of hospital admissions for chickenpox in children: an Italian multicentre study in the pre-vaccine era. Acta Paediatr. 2007;96:1490-3.

96. Nilsson A, Ortqvist A. Severe varicella pneumonia in adults in Stockholm County 1980-1989. Scand J Infect Dis. 1996:28:121-3.

97. Pena-Rey I, Martinez de Aragon MV, Villaverde Hueso A, Terres Arellano M, Alcalde Cabero E, Suarez Rodriguez B. Epidemiology of varicella in spain pre-and post-vaccination periods. Rev Esp Salud Publica. 2009;83:711-24.

98. Perez-Yarza EG, Arranz L, Alustiza J, et al. Hospital admissions for varicella complications in children aged less than 15 years old. An Pediatr (Barc). 2003:59:229-33.

99. Pierik JG, Gumbs PD, Fortanier SA, Van Steenwijk PC, Postma MJ. Epidemiological characteristics and societal burden of varicella zoster virus in the Netherlands. BMC Infect Dis. 2012;12:110. 
100. Rack AL, Grote V, Streng A, et al. Neurologic varicella complications before routine immunization in Germany. Pediatr Neurol. 2010;42:40-8.

101. Rogalska J, Paradowska-Stankiewicz I. Chickenpox in Poland in 2012. Przegl Epidemiol. 2014:68:201-4. 323-204

102. Schurink-van't Klooster TM, de Melker HE. The National Immunisation Programme in the Netherlands; Surveillance and developments in 20142015. Bilthoven: Rijksinstituut voor Volksgezondheid en Milieu (RIVM); 2015 (RIVM rapport 2015-0134). Available from: http://www.rivm.nl/bibliotheek/ rapporten/2015-0134.pdf.

103. Theodoridou M, Laina I, Hadjichristodoulou C, Syriopoulou V. Varicellarelated complications and hospitalisations in a tertiary pediatric medical center before vaccine introduction. Eur J Pediatr. 2006;165:273-4.

104. van Lier A, van Erp J, Donker GA, van der Maas NA, Sturkenboom MC, de Melker HE. Low varicella-related consultation rate in the Netherlands in primary care data. Vaccine. 2014;32:3517-24.

105. Réseau Sentinelles. Bilan annuel 2008-2014. Available from: https:// websenti.u707.jussieu.fr/sentiweb/?rub=39.

106. Ciofi Degli Atti ML, Salmaso S, Bella A, et al. Pediatric sentinel surveillance of vaccine-preventable diseases in Italy. Pediatr Infect Dis J. 2002;21:763-8.

107. Fornaro P, Gandini F, Marin M, et al. Epidemiology and cost analysis of varicella in Italy: results of a sentinel study in the pediatric practice. Italian sentinel group on pediatric infectious diseases. Pediatr Infect Dis J. 1999;18:414-9.

108. Iseli A, Aebi C, Banz K, Brunner M, Schmutz AM, Heininger U. Prospective surveillance of varicella-zoster virus infections in an out-patient setting in Switzerland. Hum Vaccin. 2009;5:843-6.

109. Lowe GL, Salmon RL, Thomas DR, Evans MR. Declining incidence of chickenpox in the absence of universal childhood immunisation. Arch Dis Child. 2004;89:966-9.

110. Nicolosi A, Sturkenboom M, Mannino S, Arpinelli F, Cantarutti L, Giaquinto C. The incidence of varicella: correction of a common error. Epidemiology. 2003;14:99-102.

111. Paul E, Thiel T. Epidemiology of varicella zoster infection. Results of a prospective study in the Ansbach area. Hautarzt. 1996;47:604-9.

112. Perez-Farinos N, Ordobas M, Garcia-Fernandez C, et al. Varicella and herpes zoster in Madrid, based on the sentinel general practitioner network: 19972004. BMC Infect Dis. 2007:7:59.

113. Ross AM, Fleming DM. Chickenpox increasingly affects preschool children. Commun Dis Public Health. 2000:3:213-5.

114. Sabbe M, Braeye T, Hue D, Grammens T, Qouilin S. Maladies infectieuses pediatriques a prevention vaccinale. Tendances et developpements en Belgique et dans les Communautes, 2012. Institut Scientifique de Sante Publique. Available from: https://epidemio.wiv-isp.be/ID/diseases/ DocumentsNPD_Rapport_2012_FR.pdf.

115. Sabbe M, Vanthomme K, Top G, Van Casteren V. Epidemiologie van windpokken en zona vastgesteld door huisartsen: 2006-2010. Vlaams Infektieziekbulletin 82/2012/4. Available from: https://www.zorg-engezondheid.be/sites/default/files/atoms/files/2012-4_82_Epidemiologie_ zona_windpok_M.\%20Sabbe.pdf.

116. Stefler D, Bhopal R. Comparison of the Hungarian and Scottish communicable disease control systems: lessons for a convergent European Community. Public Health. 2010;124:167-73.

117. Urtiaga M, Irisarri F, Zabala A. Diseases of compulsory notification (DCN) in Navarra. 2002. An Sist Sanit Navar. 2003:26:99-108.

118. INSERM. Centre d'epidémiologie sur les causes médicales de décès (CépiDC): Base de données sur les causes de décès. Available from: http:// www.cepidc.inserm.fr/inserm/html/index2.htm.

119. Cameron JC, Allan G, Johnston F, Finn A, Heath PT, Booy R. Severe complications of chickenpox in hospitalised children in the UK and Ireland. Arch Dis Child. 2007;92:1062-6.

120. Grote V, von Kries R, Springer W, Hammersen G, Kreth HW, Liese J. Varicellarelated deaths in children and adolescents-Germany 2003-2004. Acta Paediatr. 2008:97:187-92.

121. Lantto M, Renko M, Uhari M. Changes in infectious disease mortality in children during the past three decades. Pediatr Infect Dis J. 2013;32:e355-9.

122. EUVAC.NET. Varicella surveillance report 2008-2009. Available from: http:// ecdc.europa.eu/en/publications/Publications/varicella_report_2008_2009_ euvacnet.pdf.

123. EUVAC.NET. Varicella surveillance report 2010. Available from: http://ecdc europa.eu/en/publications/Publications/varicella_report_2010_euvacnet.pdf.

124. Arama V Rafila A Streinu-Cercel A, et al Varicella in Romania: epidemiological trends, 1986-2004. Euro Surveill. 2005;10:E050811.050816.
125. Baldo V, Baldovin T, Russo F, et al. Varicella: epidemiological aspects and vaccination coverage in the Veneto region. BMC Infect Dis. 2009;9:150.

126. Lipke M, Paradowska-Stankiewicz I. Chickenpox in Poland in 2011. Przegl Epidemiol. 2013;67:195-7. 317-198

127. Wutzler P, Neiss A, Banz K, Goertz A, Bisanz H. Can varicella be eliminated by vaccination? Potential clinical and economic effects of universal childhood varicella immunisation in Germany. Med Microbiol Immunol. 2002;191:89-96.

128. Baldo V, Ferro A, Napoletano G, et al. Universal varicella vaccination in the Veneto region, Italy: launch of a programme targeting all children aged 14 months and susceptible adolescents. Euro Surveill. 2007;12:E071101 071103.

129. Munoz MP, Dominguez A, Salleras L. Estimated varicella incidence on the basis of a seroprevalence survey. Epidemiol Infect. 2001;127:501-7.

130. Ziebold C, von Kries R, Lang R, Weigl J, Schmitt HJ. Severe complications of varicella in previously healthy children in Germany: a 1-year survey. Pediatrics. 2001;108:E79.

131. Wolleswinkel-van den Bosch JH, Speets AM, Rumke HC, Gumbs PD, Fortanier SC. The burden of varicella from a parent's perspective and its societal impact in The Netherlands: an internet survey. BMC Infect Dis. 2011;11:320.

132. Saddier P, Floret D, Guess HA, et al. Cost of varicella in France: a study in day care centers. J Infect Dis. 1998;178(Suppl 1):S58-63.

\section{Submit your next manuscript to BioMed Central and we will help you at every step:}

- We accept pre-submission inquiries

- Our selector tool helps you to find the most relevant journal

- We provide round the clock customer support

- Convenient online submission

- Thorough peer review

- Inclusion in PubMed and all major indexing services

- Maximum visibility for your research

Submit your manuscript at www.biomedcentral.com/submit

) Biomed Central 